\title{
A NOTE ON SEMIGROUPS OF OPERATORS ON A LOCALLY CONVEX SPACE
}

\author{
K. SINGBAL-VEDAK
}

1. The object of this note is to generalize certain results in the theory of semigroups of continuous linear operators on a Banach space to the case where, instead of a Banach space, we consider a locally convex space. A family $\{T(\xi)\}_{\xi>0}$ of linear operators on a vector space is called a semigroup if $T(\xi+\eta)=T(\xi) \circ T(\eta), \xi$, $\eta \in(0, \infty)$. E. Hille [4] and N. Dunford [2] have proved that if $\{T(\xi)\}_{\xi>0}$ is a semigroup of bounded linear operators on a Banach space $E$ such that for every $x \in E, \xi \rightarrow T(\xi) x$ is a measurable function from $(0, \infty)$ into $E$ and such that $\|T(\xi)\|_{\delta \leq \xi \leq 1 / \delta}$ is bounded for every $\delta>0(\delta<1)$, then $\xi \rightarrow T(\xi) x$ is a continuous function from $(0, \infty)$ into $E$ for $x \in E$. Proposition 2 is an analogue of this result while Propositions 3 and 4 are analogues of results due to R. S. Phillips [5] and P. Lax, respectively.

2. Definition 1 . Let $E$ be a locally convex space, $S$ a set and $\mathfrak{M}$ a $\sigma$-ring of "measurable" subsets of $S$. A function $x: S \rightarrow E(\xi \rightarrow x(\xi))$ is called measurable if it is the limit, almost everywhere, of a sequence of countably valued functions. (A function is said to be countably valued if its range is countable and it takes each value different from zero on a measurable set.)

REMARK. If $x(\xi)$ is a measurable $E$-valued function and $p$ is any continuous seminorm on $E$, then $p(x(\xi))$ is a real-valued measurable function on $S$.

Proposition 1. Let $S=(0, \infty)$ with $\mathfrak{M}$ the $\sigma$-ring of Lebesgue measurable subsets of $(0, \infty)$ and $x(\sigma)$ be a measurable function from $(0, \infty)$ into $E$. Then for any continuous seminorm $q$ on $E$, there exists a sequence $\left\{x_{n}(\sigma)\right\}_{n=1,2,}$. of countably valued functions such that $q\left(x(\sigma)-x_{n}(\sigma)\right)$ $\rightarrow 0$ as $n \rightarrow \infty$ uniformly for $\sigma$ outside a set of measure zero.

We shall prove the proposition under a weaker hypothesis, viz., that $x(\sigma)$ is weakly measurable (i.e., for every continuous linear functional $x^{\prime}$ on $E\left\langle x(\sigma), x^{\prime}\right\rangle$ is a measurable function of $\sigma$ on $\left.(0, \infty)\right)$ and that $x(\sigma)$ is almost separably valued (i.e., $x(\sigma)$ belongs to a separable subspace of $E$ almost everywhere).

We may suppose that the range of $x(\sigma)$ is contained in a separable subspace $L$ of $E$ and find a sequence $\left\{x_{n}(\sigma)\right\}_{n \geq 1}$ of countably valued

Received by the editors December 30, 1963. 
functions such that $q\left(x_{n}(\sigma)-x(\sigma)\right)$ tends to zero as $n$ tends to infinity uniformly for $\sigma$ in $(0, \infty)$. Let $\tilde{L}$ denote the quotient space of $L$, on

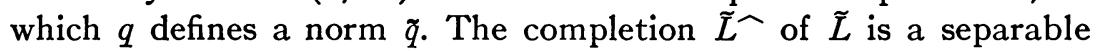
Banach space. Hence its conjugate space $\left(\tilde{L}^{\widehat{ }}\right)^{\prime}$ contains a sequence $\left\{y_{n}^{*}\right\}_{n=1,2}, \ldots$ such that

$$
\tilde{q}(\tilde{x})=\sup _{n}\left|\left\langle\tilde{x}, y_{n}^{*}\right\rangle\right| \text { for } \bar{x} \in \tilde{L} \widehat{C} .
$$

Now $x \rightarrow y_{n}^{*}(\tilde{x})$, where $\tilde{x}$ is the image of $x$ by the quotient map $L \rightarrow \tilde{L}$, is a continuous linear functional on $L$. By the Hanh-Banach theorem, there exists a continuous linear functional $x_{n}^{\prime}$ on $E$ such that

$$
\left\langle\tilde{x}, y_{n}^{*}\right\rangle=\left\langle x, x_{n}^{\prime}\right\rangle \text { for } x \in L \text { and } n=1,2, \cdots \text {. }
$$

Now $q(x(\sigma))=\tilde{q}\left[(x(\sigma))^{\sim}\right]=\sup _{n}\left|\left\langle x(\sigma), x_{n}^{\prime}\right\rangle\right|$ is measurable as $\left\langle x(\sigma), x_{n}^{\prime}\right\rangle$ is measurable for $n=1,2, \ldots$. Similarly, for any $a \in L$, $q(x(\sigma)-a)$ is a measurable function. Let $A=\{\sigma \mid q(x(\sigma))>0\}$. If $\left\{a_{i}\right\}$ is a sequence dense in $L$ and $A_{i}=A \cap\left\{\sigma \mid q\left(x(\sigma)-a_{i}\right)\right\}<1 / n$ then $A=\bigcup_{i=1}^{\infty} A_{i}$. Let $B_{1}=A_{1}, B_{j}=A_{j}-\bigcup_{i \leqslant j-1} B_{i}(j>1)$. The $B_{i}$ are disjoint measurable and $\mathrm{U} B_{i}=\bigcup A_{i}=A$.

Let

$$
\begin{aligned}
x_{n}(\sigma) & =a_{i} \quad \text { for } \sigma \in B_{i}, \\
& =0 \quad \text { for } \sigma \notin A .
\end{aligned}
$$

Then $q\left(x(\sigma)-x_{n}(\sigma)\right)<1 / n$ for all $\sigma$ so that $q\left(x(\sigma)-x_{n}(\sigma)\right) \rightarrow 0$ as $n \rightarrow \infty$ uniformly for $\sigma$ in $(0, \infty)$.

Definition 2. A semigroup of operators on a locally convex space $E$ is said to be measurable if, for $x \in E$, the function $\xi \rightarrow T(\xi) x$ is measurable from $(0, \infty)$ to $E$.

Proposition 2. If $\{T(\xi)\}_{\xi>0}$ is a measurable semigroup of continuous linear operators in a locally convex space $E$ such that, for every $[\alpha, \beta] \subset(0, \infty),\{T(\xi)\}_{\alpha \leq \xi \leq \beta}$ is an equicontinuous family of operators, then $\xi \rightarrow T(\xi) x$ is continuous for every $x \in E$.

Proof. We have to show that for $\xi \in(0, \infty)$ and $x \in E$,

$$
T(\xi \pm \eta) x-T(\xi) x \rightarrow 0 \quad \text { in } E \text { as } \eta \rightarrow 0 .
$$

Let $0<\alpha<\beta<\xi$. As $\{T(\tau)\}_{\tau \in[\alpha, \beta]}$ is an equicontinuous set, given any continuous seminorm $p$ on $E$, there exists a continuous seminorm $q$ on $E$ and $k>0$ such that

$$
p(T(\tau) y) \leqq k q(y) \quad \text { for } \alpha \leqq \tau \leqq \beta \text { and } y \in E .
$$

Let $\eta_{0}>0$ be such that $\alpha-\eta>0$ and $\beta<\xi-\eta$ for $0<\eta<\eta_{0}$. By (2), we have 


$$
\begin{aligned}
p(T(\xi \pm \eta)-T(\xi) x) & =p(T(\tau)[T(\xi \pm \eta-\tau) x-T(\xi-\tau) x]) \\
& \leqq k q(T(\xi \pm \eta-\tau) x-T(\xi-\tau) x) .
\end{aligned}
$$

As $\{T(\xi \pm \eta-\tau)\}_{\tau \in[\alpha, \beta]}$ and $\{T(\xi-\tau)\}_{\tau \in[\alpha, \beta]}$ are equicontinuous sets, $\{T(\xi \pm \eta-\tau) x\}_{\tau \in[\alpha, \beta]}$ and $\{T(\xi-\tau) x\}_{\tau \in[\alpha, \beta]}$ are bounded subsets of $E$ so that $q(T(\xi \pm \eta-\tau) x-T(\xi-\tau) x)$ is a bounded measurable function of $\tau$ in $[\alpha, \beta]$. Integrating (3) with respect to $\tau$ from $\alpha$ to $\beta$ we get

$$
(\beta-\alpha) p(T(\xi \pm \eta) x-T(\xi) x)
$$

$$
\leqq k \int_{\alpha}^{\beta} q(T(\xi \pm \eta-\tau) x-T(\xi-\tau) x) d \tau .
$$

The integral on the right-hand side tends to zero with $\eta$ if we show that, for given $\epsilon>0$, there exists a continuous $E$-valued function $f_{\mathbf{c}}(\tau)$ such that

$$
\int_{\alpha-\eta_{0}}^{\beta+\eta_{0}} q\left(T(\xi-\tau) x-f_{\epsilon}(\tau)\right) d \tau<\epsilon .
$$

For then

$$
\begin{aligned}
& \int_{\alpha}^{\beta} q(T(\xi \pm \eta-\tau) x-T(\xi-\tau) x) d \tau \\
& \quad \leqq \int_{\alpha}^{\beta} q\left(T(\xi \pm \eta-\tau) x-f_{\epsilon}(\tau \mp \eta)\right) d \tau+\int_{\alpha}^{\beta} q\left(f_{\epsilon}(\tau \mp \eta)-f_{\epsilon}(\tau)\right) d \tau \\
& \quad+\int_{\alpha}^{\beta} q\left(f_{\epsilon}(\tau)-T(\xi-\tau) x\right) d \tau
\end{aligned}
$$

and the first and the third integral are each majorised by $\epsilon$ and the second integral tends to zero with $\eta$ since $f_{\epsilon}(\tau)$ is continuous. Now $h(\tau)=T(\xi-\tau) x$ being measurable from proposition (1), it follows that, given $\epsilon>0$, there exists a countably valued function $x(\tau)$ such that $\int_{\alpha-\eta_{0}}^{\beta+\eta_{0}} q(h(\tau)-x(\tau)) d \tau<\epsilon / 3$. Let $x(\tau)=\sum_{i=1}^{\infty} a_{i} \chi_{A_{i}}$, where $a_{i} \in E$ and $\chi_{A_{i}}$ are characteristic functions of disjoint measurable sets $A_{i}$ contained in $\left[\alpha-\eta_{0}, \beta+\eta_{0}\right]$. Then we can choose $m$ such that for $y(\tau)$ $=\sum_{i=1}^{m} a_{i} \chi_{A_{i}}, \quad \int_{\alpha-\eta_{0}}^{\beta+\eta_{0}} q(x(\tau)-y(\tau)) d \tau<\epsilon / 3$. Let $q(y(\tau))<M$. Given $\delta>0$ we can find compact sets $K_{i} \subset A_{i}$ and open sets $O_{i} \supset A_{i}(i=1,2, \cdots, m)$ such that $\sum_{i=1}^{m} m\left(O_{i}-K_{i}\right)<\delta(m$ being Lebesgue measure). For every $i$ there exists a continuous function $g_{i}(\tau), 0 \leqq g_{i}(\tau) \leqq 1$, such that $g_{i}(\tau)$ equals 1 on $K_{i}$ and 0 outside $O_{i}$. If $g(\tau)=\sum_{i=1}^{m} a_{i} g_{i}(\tau)$, then $q(g(\tau))<m M$ and $g(\tau)=y(\tau)$ for $\tau \notin \bigcup_{i=1}^{m}\left(O_{i}-K_{i}\right)$ so that $\int_{\alpha-\eta_{0}}^{\beta+\eta_{0}} q(y(\tau)-g(\tau)) d \tau<2 m M \delta<\epsilon / 3$ for $\delta<\epsilon / 6 m M$. Taking $f_{\epsilon}(\tau)=g(\tau)$, (5) is satisfied. 
REMARK 1. Proposition 2 remains true if instead of the hypothesis that $\xi \rightarrow T(\xi) x$ are $E$-valued measurable functions for $x \in E$, we have the weaker hypothesis that these functions are weakly measurable and almost separably valued. For, from the proof of Proposition 1, given $\epsilon>0$, there exists a countably valued function $x(\tau)$ such that $\int_{\alpha-\eta_{0}}^{\beta+\eta_{0}} q[h(\tau) x-x(\tau)] d \tau<\epsilon / 3$ and the proof is completed by the same method as above.

REMARK 2. The converse of Proposition 2, viz., the statement that "If $\{T(\xi)\}_{\xi>0}$ is a semigroup such that $\xi \rightarrow T(\xi) x$ are continuous functions for $x \in E$, then $\{T(\xi)\}_{\xi>0}$ is a measurable semi-group such that for every $[\alpha, \beta] \subset(0, \infty),\{T(\xi)\}_{\xi \in[\alpha, \beta]}$ is an equicontinuous set of maps of $E$ into $E$ " is true if $E$ is barrelled (tonnelé) or is the strong dual of a metrisable locally convex space. For, if $\xi \rightarrow T(\xi) x$ is continuous, then it is measurable and maps the compact set $[\alpha, \beta] \subset(0, \infty)$ onto a compact subset of $E$. Thus $\{T(\xi)\}_{\alpha_{\S} \xi \beta}$ is compact in $\mathfrak{\Omega}_{s}(E, E)$, the space of linear continuous maps of $E$ into $E$ furnished with the topology of simple convergence, and therefore is a closed bounded subset of $\mathfrak{R}_{8}(E, E)$. If $E$ is barrelled, this implies that $\{T(\xi)\}_{\xi \in[\alpha, \beta]}$ is equicontinuous. If $E$ is the strong dual of a metrisable space, let $\left(\xi_{n}\right)_{n=1,2}, \ldots$ denote the sequence of rationals in $[\alpha, \beta]$. Then $\left\{T\left(\xi_{n}\right)\right\}_{n=1,2, \ldots}$ being a subset of $\{T(\xi)\}_{\alpha \leq \xi \leq \beta}$ is bounded in $\Omega_{s}(E, E)$ and is therefore equicontinuous [3, Proposition 1, p. 62]. Hence its closure in $\mathfrak{\Omega}_{s}(E, E)$ is equicontinuous. Now, for any $\sigma \in[\alpha, \beta]$, there exists a subsequence $\left(\xi_{n_{k}}\right)$ of $\left(\xi_{n}\right)$ such that $\xi_{n_{k}} \rightarrow \sigma$ as $k \rightarrow \infty$, so that $T(\sigma) x=\lim _{k \rightarrow \infty} T\left(\xi_{n_{k}}\right) x$ for every $x \in E$. Thus $\{T(\xi)\}_{\alpha \leq \xi \leq \beta}$ is the closure of $\left\{T\left(\xi_{n}\right)\right\}_{n=1,2}, \ldots$ in $\mathfrak{R}_{8}(E, E)$ which is equicontinuous.

Proposition 3. Let $\{T(\xi)\}_{\xi>0}$ be a measurable semigroup of continuous linear operators in a Fréchet space $E$. Then for any $[\alpha, \beta]$ $C(0, \infty)$,

$$
\{T(\xi)\}_{\alpha \leq \xi \leq \beta}
$$

is an equicontinuous set of maps of $E$ into $E$.

Proof. Let $\left\{p_{n}\right\}$ be a sequence or seminorms on $E$ defining the topology of $E$. Since $E$ is Fréchet, it is sufficient to prove that $\{T(\xi) x\}_{\xi \in[\alpha, \beta]}$ is bounded in $E$ for any fixed $x \in E$. If this is not true, there exists an $x \in E$ and an integer $i_{0}$ and a sequence $\left\{\xi_{n}\right\}_{n=1,2}, \ldots$ tending to a real number $\gamma$ where $\xi_{n}, \gamma \in[\alpha, \beta]$, such that

$$
p_{i_{0}}\left(T\left(\xi_{n}\right) x\right) \geqq n \text { for all } n \text {. }
$$

Let $\left\{F_{i}\right\}_{i=1,2}, \ldots$ be a sequence of measurable subsets of $(0, \gamma]$ such that 
(i) $F_{i+1} \subset F_{i}$,

(ii) the measure $m\left(F_{i}\right)$ of $F_{i}>\gamma / 2+\gamma / 3 i$,

(iii) the measurable function $p_{i}(T(\xi) x)$ is bounded on $F_{i}$ by $M_{i}$, say.

Clearly such a sequence exists and $F=\bigcap_{i=1}^{\infty} F_{i}$ is measurable with $m(F) \geqq \gamma / 2$ and $p_{i}\{T(\xi) x\} \leqq M_{i}$ for $\xi \in F$, and $i=1,2, \cdots$, i.e., $\{T(\xi) x\}_{\xi \in F}$ is a bounded subset of $E$.

The sets

$$
A_{n}=\left\{\xi_{n}-\eta \mid \eta \in F \cap\left(0, \xi_{n}\right)\right\}, \quad n=1,2, \cdots
$$

are measurable and

$$
m\left(A_{n}\right) \geqq \frac{\gamma}{4} \text { for } n \geqq N, \text { say. }
$$

For $\eta \in F \cap\left(0, \xi_{n}\right)$,

$$
n=p_{i_{0}}\left[T\left(\xi_{n}\right) x\right] \leqq p_{i_{0}}\left[T\left(\xi_{n}-\eta\right) T(\eta) x\right] .
$$

Let $\sigma \in A_{n}$ be arbitrary. Then

so that

$$
\sigma=\xi_{n}-\eta \text { for some } \eta \in F \cap\left(0, \xi_{n}\right),
$$

$$
p_{i_{0}}[T(\sigma) T(\eta) x] \geqq n .
$$

Let $A=\lim \sup _{n \rightarrow \infty} A_{n}$. Then $m(A) \geqq \gamma / 4$ by (1). For $\sigma_{0} \in A, p_{i_{0}}$ is unbounded on $T\left(\sigma_{0}\right)\left[\{T(\eta) x\}_{\eta \in F}\right]$ by (2), i.e., $T\left(\sigma_{0}\right)\left[\{T(\eta) x\}_{\eta \in F}\right]$ is not a bounded subset of $E$. This is a contradiction since $T\left(\sigma_{0}\right)$ is a continuous linear map of $E$ into $E$ and $\{T(\eta) x\}_{\eta \in F}$ is a bounded set in $E$.

Combining Propositions 2 and 3 we have the

THEOREM. Every measurable semigroup of continuous linear operators on a Fréchet space is a continuous function from $(0, \infty)$ into the space of continuous linear maps of $E$ into $E$ furnished with the topology of simple convergence.

3. Proposition 4. Let $\{T(\xi)\}_{\xi>0}$ be a measurable semigroup of continuous linear operators on a locally convex space $E$ such that $\{T(\xi)\}_{\alpha \leq \xi \leq \beta}$ is an equicontinuous family for every $[\alpha, \beta] \subset(0, \infty)$ and such that for some $\xi_{0}>0, T\left(\xi_{0}\right)$ is a combact operator on $E$. Then, for any $a>\xi_{\text {n. }}$. $T(a)$ is a compact operator and $\xi \rightarrow T(\xi)$ is a continuous mapping of $(a, \infty)$ into $\Omega_{\mathscr{S}}(E, E)$ where $\mathbb{R}_{\mathfrak{S}}(E, E)$ is the space of continuous linear maps of $E$ into $E$ furnished with the topology of uniform convergence on bounded subsets of $E$. 
Proof. Let $b>a>\xi_{0}$ and let $B$ be a bounded subset of $E, V$ a neighbourhood of 0 in $E$ and

$$
W(B, V)=\left\{u \in \mathcal{L}_{S}(E, E) \mid u(B) \subset V\right\} .
$$

We shall show that there exists $\delta>0$ such that

$$
T(\xi+\eta)-T(\xi) \in W(B, V)
$$

for $|\eta|<\delta, a<\xi<b, a<\xi+\eta<b$.

As the sets $\{W(B, V)\}$, where $B$ is a bounded subset of $E$ and $V$ is a neighbourhood of 0 in $E$, form a fundamental system of neighbourhoods of zero in $\Omega_{\varsigma}(E, E)$, this will prove that $T(\xi+\eta)-T(\xi) \rightarrow 0$ in $\mathcal{R}_{\odot}(E, E)$ as $\eta \rightarrow 0$. Let $V_{1}$ be a neighbourhood of 0 in $E$ such that

$$
V_{1}+V_{1}+V_{1} \subset V \text {. }
$$

Let $V_{2}$ be a neighbourhood of 0 in $E$ such that

$$
V_{2} \subset V_{1}
$$

and

$$
T\left(\xi-\xi_{0}\right) V_{2} \subset V_{1} \text { for } a \leqq \xi \leqq b .
$$

This is possible since $\left\{T\left(\xi-\xi_{0}\right)\right\}_{a \leq \xi_{\leq} b}$ is an equicontinuous family of maps of $E$ into $E$.

Now, $T\left(\xi_{0}\right)$ being a compact operator, there exists a neighbourhood $U$ of 0 such that $T\left(\xi_{0}\right) U$ is a relatively compact subset of $E$. The bounded subset $B$ of $E$ is absorbed by $U$, so that $T\left(\xi_{0}\right) B$ is also relatively compact. Given a neighbourhood $V_{2}$ of 0 in $E$, there exist $x_{1}, x_{2}, \cdots, x_{n} \in E$ such that

$$
T\left(\xi_{0}\right) B \subset \bigcup_{k=1}^{n}\left\{T\left(\xi_{0}\right) x_{k}+V_{2}\right\}
$$

i.e., for any $x \in B$, there exists an $x_{k}$ such that

$$
T\left(\xi_{0}\right) x-T\left(\xi_{0}\right) x_{k} \in V_{2} .
$$

By Proposition 1, for any $x \in E, \xi \rightarrow T(\xi) x$ is a continuous function from $(0, \infty)$ into $E$. In particular, $\xi \rightarrow T(\xi) x_{k}$ are continuous functions for $k=1,2, \cdots, n$. We can, therefore, find $\delta>0$ such that, for $k=1,2, \cdots, n$,

$$
T(\xi+\eta) x_{k}-T(\xi) x_{k} \in V_{2} \text { if }|\eta|<\delta, a \leqq \xi, \xi+\eta \leqq b .
$$

Let $x \in B$ and $x_{k}$ be as in (4). Then 


$$
\begin{aligned}
T(\xi+\eta) x-T(\xi) x= & T(\xi+\eta) x-T(\xi+\eta) x_{k}+T(\xi+\eta) x_{k}-T(\xi) x_{k} \\
& +T(\xi) x_{k}-T(\xi) x \\
= & T\left(\xi+\eta-\xi_{0}\right)\left[T\left(\xi_{0}\right) x-T\left(\xi_{0}\right) x_{k}\right] \\
& +\left[T(\xi+\eta) x_{k}-T(\xi) x_{k}\right] \\
& +T\left(\xi-\xi_{0}\right)\left[T\left(\xi_{0}\right) x_{k}-T\left(\xi_{0}\right) x\right] \\
\in & T\left(\xi+\eta-\xi_{0}\right) V_{2}+V_{2}+T\left(\xi-\xi_{0}\right) V_{2} \\
\subset & V_{1}+V_{1}+V_{1} \subset V
\end{aligned}
$$

i.e., $[T(\xi+\eta)-T(\xi)] B \subset V$ for $|\eta|<\delta, a \leqq \xi+\eta \leqq b, \xi_{0}<a$. This proves the required continuity of the function $\xi \rightarrow T(\xi)$.

That $T(\xi)$ is compact for $\xi>\xi_{0}$ follows from the fact $T\left(\xi_{0}\right)$ is a compact operator and $T(\xi)=T\left(\xi-\xi_{0}\right) T\left(\xi_{0}\right)$ where $T\left(\xi-\xi_{0}\right)$ is a continuous linear map of $E$ into $E$.

\section{REFERENCES}

1. N. Bourbaki, Espaces vectoriels topologiques, Hermann, Paris, 1955.

2. N. Dunford, On one parameter groups of linear transformations, Ann. of Math. (2) 39 (1938), 567-573.

3. A. Grothendieck, Sur les espaces $(F)$ et $(D F)$, Summa Brasil. Math. 3 (1954), $57-121$.

4. E. Hille, Functional analysis and semigroups, Amer. Math. Soc. Colloq. Publ. Vol. 31, Amer. Math. Soc., Providence, R. I., 1948.

5. R. S. Phillips, On one parameter semi-groups of linear transformatiuns, Proc. Amer. Math. Soc. 2 (1951), 234-237.

Tata Institute of Fundamental Research, Bombay, India

\section{ON RECURSIVELY DEFINED ORTHOGONAL POLYNOMIALS ${ }^{1}$}

\section{T. S. CHIHARA}

1. Introduction. Consider a set $\left\{P_{n}(x)\right\}$ of orthogonal polynomials defined by the classical recurrence formula,

$$
\begin{array}{rlrl}
P_{n}(x) & =\left(x-c_{n}\right) P_{n-1}(x)-\lambda_{n} P_{n-2}(x) & & (n=1,2,3, \cdots), \\
P_{-1}(x) & =0, \quad P_{0}(x)=1, \quad c_{n} \text { real, } & \lambda_{n+1}>0 .
\end{array}
$$

In [2], the author initiated a study of (1.1) based on the chain sequences of Wall [6], the fundamental relation being that the zeros

Presented to the Society, January 26, 1964; received by the editors October 10, 1963.

${ }^{1}$ This work was supported by the National Science Foundation (NSF-GP 1230). 